\title{
Epiretinal Membrane Peeling in Eyes with Retinal Vein Occlusion: Visual and Morphologic Outcomes
}

\author{
Maria Vittoria Cicinelli (D) · Irini Chatziralli · Sara Touhami • \\ Anissa Smaoui · Beatrice Tombolini · Marco Nassisi · Panagiotis Theodossiadis • \\ Rosangela Lattanzio · Francesco Bandello
}

Received: November 16, 2021 / Accepted: January 10, 2022 / Published online: January 31, 2022

(C) The Author(s) 2022

\section{ABSTRACT}

Introduction: To evaluate the anatomical and functional outcomes of pars plana vitrectomy (PPV) and epiretinal membrane (ERM) peeling in patients with retinal vein occlusion (RVO) and secondary ERM.

Supplementary Information The online version contains supplementary material available at https:// doi.org/10.1007/s40123-022-00461-7.

M. V. Cicinelli · F. Bandello

School of Medicine, Vita-Salute San Raffaele

University, Milan, Italy

M. V. Cicinelli $(\bowtie) \cdot$ B. Tombolini - R. Lattanzio · F. Bandello

Department of Ophthalmology, University Vita-

Salute, IRCCS Ospedale San Raffaele, via Olgettina 60, 20132 Milan, Italy

e-mail: cicinelli.mariavittoria@hsr.it

B. Tombolini

e-mail: tombolini.beatrice@hsr.it

R. Lattanzio

e-mail: lattanzio.rosangela@hsr.it

F. Bandello

e-mail: bandello.francesco@hsr.it

I. Chatziralli · P. Theodossiadis

2nd Department of Ophthalmology, National and Kapodistrian University of Athens, Athens, Greece e-mail: eirchat@yahoo.gr
Methods: Retrospective, multicenter study including patients with RVO and ERM who underwent PPV and ERM peeling with or without phacoemulsification. Demographic, clinical, surgical, and optical coherence tomography (OCT) features were recorded at the time of ERM peeling (baseline). Best-corrected visual acuity (BCVA) and central macular thickness (CMT)

P. Theodossiadis

e-mail: patheo@med.uoa.gr

S. Touhami · A. Smaoui

Department of Ophthalmology, Lariboisière

University Hospital, Université de Paris, Paris, France

S. Touhami · A. Smaoui

Department of Ophthalmology, Pitié Salpêtrière

University Hospital, Sorbonne Université, Paris,

France

e-mail: sara.touhami@aphp.fr

A. Smaoui

e-mail: smaoui.anissa@yahoo.fr

M. Nassisi

Ophthalmological Unit, Fondazione IRCCS Ca'

Granda, Ospedale Maggiore Policlinico, Milan, Italy

M. Nassisi

Department of Clinical Sciences and Community

Health, University of Milan, Milan, Italy

e-mail: m.nassisi@gmail.com 
were longitudinally collected up to 36 months after surgery. Clinical factors associated with BCVA and CMT and disappearance of macular edema during follow-up were investigated.

Results: Twenty-one eyes of 21 patients with a median follow-up of 18 months were included. The BCVA improved significantly after ERM peeling (baseline vs. 24 months, $p=0.01$ ). Absence of the external liming membrane/ellipsoid zone on OCT was associated with worse visual outcomes (regression estimate [95\% confidence interval, CI] $=0.93$ [0.39-1.48] logMAR, $p=0.004)$. Eyes with disorganization of the inner retinal layers at baseline had higher CMT values at each visit (regression estimate $[95 \% \mathrm{CI}]=114.1[78.9-219.4] \mu \mathrm{m}, p=0.004)$. Older age at the time of RVO $(p=0.03)$ and branch RVO $(p=0.04)$ were risk factors for persistent macular edema after ERM removal.

Conclusion: PPV and ERM removal provided encouraging functional and morphological results in eyes with RVO, with disappearance of macular edema in most eyes. The integrity of the outer retina and preservation of inner retinal segmentation were associated with better visual and anatomical outcomes after ERM removal, respectively.

Keywords: Anti-vascular endothelial growth factor; Cystoid macular edema; Dexamethasone; Epiretinal membrane; Intravitreal corticosteroids; Retinal vein occlusion

\section{Key Summary Points}

Why carry out this study?

Epiretinal membrane (ERM) may exacerbate macular edema in patients with retinal vein occlusion (RVO) and may reduce drug penetration of intravitreal antiangiogenic agents. Therefore, patients with RVO may potentially benefit from surgical removal of ERM.
This study evaluated the anatomical and functional outcomes of pars plana vitrectomy (PPV) and membrane peeling in patients with RVO and secondary ERM.

\section{What was learned from the study?}

PPV and ERM removal provided functional and morphological improvements in eyes with RVO, with disappearance of macular edema in most eyes.

Irreversible damage to the retinal layers was associated with poorer outcomes. Older age and branch RVO were risk factors for persistent macular edema after ERM removal.

\section{INTRODUCTION}

Epiretinal membrane (ERM) refers to a sheetlike fibroglial cellular tissue proliferation over the internal limiting membrane (ILM) in the macular area [1]. ERM is most commonly idiopathic, developing after posterior vitreous detachment (PVD) and migration of retinal glial and retinal pigment epithelial cells into the anterior retinal surface. Secondary ERM has been described in retinal vasculopathies, intraocular inflammation, retinal detachment, retinal surgeries, and ocular trauma [2]. Secondary ERM may be an additive cause of visual loss in eyes with underlying ocular diseases.

Retinal vein occlusion (RVO), either central RVO (CRVO) or branch RVO (BRVO), is the second most common retinal vascular disease after diabetic retinopathy, and it may lead to irreversible visual loss if left untreated [3]. The prevalence of ERMs in patients with RVO ranges between 14 and $16 \%$, but it is likely underestimated [4-6]. ERM may exacerbate macular edema in patients with RVO, may reduce drug penetration of intravitreal antiangiogenic agents, and may eventually progress to a pseudohole, or more rarely, a macular hole. 
Therefore, patients with RVO may potentially benefit from surgical removal of ERM.

Previous studies have examined the outcomes of secondary ERM surgical removal in eyes with tractional diabetic macular edema, reporting good anatomical and variable functional results $[7,8]$. In addition, pars plana vitrectomy (PPV) has been proven effective in ERM due to uveitis or trauma $[9,10]$. Limited data exist on eyes with RVO and coexistent ERM undergoing PPV and ERM peeling; these studies had a relatively short follow-up and included eyes with either CRVO or BRVO [11, 12].

Based on these observations, the purpose of this study was to evaluate the anatomical and functional outcomes of PPV and ERM peeling in patients with CRVO and BRVO and secondary ERM.

\section{METHODS}

This study is a retrospective, multicenter, observational study of patients with RVO and ERM who underwent PPV and ERM peeling with or without cataract phacoemulsification between July 2012 and January 2021. Patients were included from the San Raffaele Scientific Institute (Milano, Italy), the Second Department of Ophthalmology, the University of Athens (Athens, Greece), the Ospedale Maggiore Policlinico (Milano, Italy), and the Lariboisière University Hospital, Université de Paris (Paris, France). The study adhered to the tenets of the Declaration of Helsinki (1964) and received the approval of the local institutional review boards.

The decision to pursue PPV and ERM removal was made on a personal basis, considering each patient's history of macular edema, response to previous intravitreal therapies, expectations, and compliance to visits and treatments. Overall, ERM peeling was performed in patients with RVO and history of macular edema with a suboptimal response to previous therapeutic strategies, non-improving visual acuity, or persistence/recurrence of macular thickening despite treatments. All the eyes had evident ERM on optical coherence tomography (OCT) scans encompassing the fovea and obliterating the foveal depression. Patients with retinal diseases other than RVO (e.g., age-related macular degeneration, diabetic retinopathy), those with RVO-related complications causing irreversible visual loss (e.g., retinal detachment or neovascular glaucoma), and those with a history of intraocular surgery except for uncomplicated phacoemulsification before ERM peeling were excluded.

Patients' charts were reviewed at the time of ERM peeling (baseline) and 3, 6, 12, 24, and 36 months after surgery; a range of 2 months for each time point was allowed due to the study's retrospective nature. The following variables were recorded at baseline: age, gender, history of glaucoma, diabetes mellitus, and cardiovascular comorbidities (systemic hypertension, deep vein thrombosis, arterial occlusive disease), interval between RVO diagnosis and PPV, previous intravitreal treatments (both anti-vascular endothelial growth factor [VEGF] agents and dexamethasone [DEX] implants), and presence of macular edema. Regarding surgery, parameters collected included the date of the surgery, phacoemulsification at the time of PPV, induction of PVD, inner limiting membrane (ILM) peeling, tamponade agent at the end of PPV, and occurrence of peri- or postoperative complications. History of intravitreal injections after the surgical procedure was also recorded.

Best-corrected visual acuity (BCVA) values, measured on decimal charts, and spectral-domain OCT (SD-OCT, Spectralis HRA+OCT, Heidelberg, Germany) scans were recorded at each visit. The OCT software automatically calculated the central macular thickness (CMT). The SD-OCT scans were scrutinized by four trained graders (MVC, IC, MN, AS) for external limiting membrane (ELM)/ellipsoid zone (EZ) damage under the fovea (in case the two outer hyperreflective bands corresponding to the ELM and EZ were interrupted or absent) and the presence of disorganization of the retinal inner layers (DRIL), defined as the loss of clear tomographic boundaries between the four innermost retinal layers [13-15]. 


\section{Statistical Analysis}

Statistical calculations were carried out with the open-source R programming language. Continuous variables were reported as the median and interquartile range (IQR) or mean \pm standard deviation (SD), and categorical variables as frequency and proportions. The BCVA was converted into $\log$ MAR and used as a continuous variable; a value of $2.0 \log$ MAR was given to counting fingers, and a value of $2.3 \log$ MAR was given to hand motion [16].

As the primary outcome, the clinical factors associated with the longitudinal BCVA and CMT variation after ERM removal were investigated. For this purpose, linear mixed models with a repeated-measures design were used, in which the eye identification number was the random factor to account for multiple measures performed in the same eye, and the explored covariate was the main effect variable. The interaction between the visit and the main effect variable was included in each model. Regression estimates and 95\% confidence intervals (CI) were computed. The estimated marginal means at different time points were compared with a Bonferroni correction. The analyses were repeated separately evaluating eyes with CRVO and BRVO.

For the secondary outcome, improvement in BCVA of at least one line (i.e., reduction in BCVA of $0.1 \log$ MAR) and disappearance of macular edema on SD-OCT after ERM removal (i.e., OCT not showing any sign of intraretinal or subretinal fluid) were considered as events, and the date on which the event was first recorded in the patients' charts was collected. Kaplan-Meier survival curves were plotted, and median survival time was estimated. Since improvement in BCVA of at least one line was recorded in $95 \%$ of eyes, risk factor analysis was not performed. On the other hand, the clinical factors associated with disappearance of macular edema on SD-OCT after ERM removal were investigated with univariable Cox proportional hazard models. For each variable, the hazard ratio (HR) and 95\% CI were reported. The patients who presented without macular edema at the time of ERM removal were excluded from survival regression models.
As the tertiary outcome, the presence of macular edema, the need for and the number of intravitreal injections (either anti-VEGF or steroids), and the rate of ELM/EZ and DRIL on OCT were compared between before and after ERM removal between paired $t$ test or chi-square tests.

The cutoff point for statistical significance was set at $p<0.05$ (two-sided).

\section{RESULTS}

\section{Patients' and Eyes' Characteristics}

A total of 21 eyes of 21 patients were included; most of the patients were male $(62 \%)$, older than 50 years, with cardiovascular comorbidities (67\%). Two-thirds (67\%) of the eyes had CRVO. Eight eyes (38\%) had received peripheral laser photocoagulation prior to ERM removal. The median interval from RVO to ERM peeling was 32 months, while the median available follow-up after ERM removal was 18 months. Table 1 shows the baseline demographic and surgical characteristics of the study sample.

All eyes had a history of macular edema secondary to RVO. Sixteen eyes (76\%) had received intravitreal treatments, namely antiVEGF agents (10 eyes, 48\%) and DEX implants (12 eyes, 57\%), before undergoing ERM peeling (Table 2). In 18 eyes (86\%), the OCT closest to the surgery date showed persistence of intraretinal fluid. Ten eyes (48\%) had DRIL, and six eyes (29\%) had subfoveal ELM/EZ layers loss on SD-OCT.

\section{Surgery Characteristics}

All eyes underwent complete vitrectomy. Of the 16 phakic eyes at the time of ERM removal, 11 (69\%) underwent combined PPV with phacoemulsification and intraocular lens implantation. Vitrectomy was mostly performed with a 25-gauge technique (67\%), with PVD induction (71\%); the ILM was peeled in $86 \%$ of cases. None of the included eyes received intraoperative intravitreal triamcinolone. The surgery was uneventful in $86 \%$ of eyes; one case of 
Table 1 Baseline demographic and surgical characteristics of patients with retinal vein occlusion (RVO) undergoing epiretinal membrane (ERM) peeling

Summary statistics (median, IQR or \%)

Patients' and eyes' characteristics

Age at RVO (years)

$63(60-69)$

Age at peeling (years)

$67(64-72)$

Gender

Male

$13(62 \%)$

Female

$8(38 \%)$

Diabetes

$3(14 \%)$

Cardiovascular risk factors

$14(67 \%)$

Glaucoma

$6(29 \%)$

Pseudophakia

$5(24 \%)$

Type of RVO

Central RVO

$14(67 \%)$

Branch RVO

Peripheral laser photocoagulation

Interval RVO to ERM peeling (months)

$32(23-53)$

Duration of follow-up after ERM peeling (months)

$18(7-34)$

Surgery characteristics

Concurrent phacoemulsification

$11(69 \%)^{*}$

Gauge

23

$6(29 \%)$

25

$14(67 \%)$

27

$1(4 \%)$

PVD induction

$15(71 \%)$

ILM peeling

$18(86 \%)$

Gas tamponade $4(19 \%)$

Summary statistics are presented as median (interquartile range, IQR) or frequencies (proportions)

$P V D$ posterior vitreous detachment, $I L M$ inner limiting membrane

${ }^{*}$ Of phakic eyes before vitrectomy (i.e., 16 eyes)

intraoperative peripheral retinal tear, one posterior capsule tear, and one case of macular hole were recorded. Macular hole was repaired during PPV. The eyes were filled with gas in four (19\%) cases, with either SF6 (three eyes) or C2F6 (one eye) (Table 1). 
Table 2 Summary of intravitreal treatment received before and after epiretinal membrane (ERM) peeling

\begin{tabular}{lccc}
\hline & \multicolumn{2}{l}{ Summary statistics } & \multirow{2}{*}{$\boldsymbol{p}$ values } \\
\cline { 2 - 3 } & Before ERM peeling & After ERM peeling & \\
\hline Patients receiving anti-VEGF injections & $10(48 \%)$ & $6(29 \%)$ & 0.3 \\
Anti-VEGF doses administered & $6(4-9)$ & $1(1-2)$ & $0.02^{*}$ \\
Patients receiving DEX injections & $12(57 \%)$ & $12(57 \%)$ & 0.9 \\
DEX implants administered & $3(1-3)$ & $2(1-6)$ & 0.5 \\
Macular edema & $18(86 \%)$ & $4(19 \%)$ & $<0.001^{*}$ \\
DRIL & $10(48 \%)$ & $4(19 \%)$ & 0.1 \\
ELM/EZ loss & $6(29 \%)$ & $6(29 \%)$ & 0.9 \\
\hline
\end{tabular}

Summary statistics are presented as median (interquartile range) or frequencies (proportions) and compared with paired $t$ test or chi-square tests, respectively

$V E G F$ vascular endothelial growth factor, $D E X$ dexamethasone, DRIL disorganization of the retinal inner layers, ELM external limiting membrane, $E Z$ ellipsoid zone

*statistically significant value

\section{Visual Outcomes after ERM Removal}

The BCVA before ERM peeling was $0.87 \pm 0.62$ logMAR, ranging between hand motion and 0.22 logMAR (Table 1S). The BCVA improved after ERM removal (Fig. 1a); multiple comparisons revealed significant change from baseline to month $6(p=0.02)$ and month $24(p=0.01)$. Improvement of at least one line occurred in 20 eyes $(95 \%)$ and was recorded after a median of 1 month (range 1-6 months) (Fig. 2a).

Globally, longitudinal visual outcomes were worse in eyes with ELM/EZ loss at baseline $(p<0.001)$ and those with persistent ELM/EZ damage after peeling $(p<0.001)$. Eyes with DRIL after ERM removal also had worse vision after ERM removal $(p=0.009)$ (Table 3$)$. When these variables were combined in a multivariable model, only ELM/EZ loss was significantly associated with a worse visual outcome (regression estimate $=$ $0.93 \log$ MAR, 95\% CI 0.39-1.48, $p=0.004)$. ELM/ EZ loss post-ERM removal was not included in the multivariable model due to collinearity with outer retinal damage before ERM removal. None of the tested interactions was significant.

Similar associations were observed separately analyzing CRVO eyes (Table 2S). None of the investigated variables was statistically associated with visual acuity in BRVO cases (Table 3S).

\section{Morphologic Outcomes after ERM Removal}

The CMT before ERM peeling was $494 \pm 126.5 \mu \mathrm{m}$ (Table 1S). The retinal thickness decreased progressively after ERM peeling (Fig. 1b), being statistically significant at 6 $(p=0.03)$ and 24 months $(p=0.02)$ compared to before surgery. Eyes with DRIL at baseline (regression estimate $=114.1 \mu \mathrm{m}, \quad 95 \% \quad$ CI 078.9-219.4, $p=0.04$ ) and those with DRIL after surgery (regression estimate $=217.5 \mu \mathrm{m}$, 95\% CI 91.7-342.7, $p=0.002$ ) had higher CMT values (Table 3 ). The presence of DRIL after surgery was confirmed as a negative anatomical prognostic factor analyzing CRVO and BRVO eyes separately (Tables $2 \mathrm{~S}$ and $3 \mathrm{~S}$ ).

Of the 18 eyes with macular edema at the time of the surgery, 14 eyes (78\%) achieved a dry macula after ERM peeling $(p<0.001)$. The median time for macular edema disappearance was 6 months (range 1-24), while the median survival time (i.e., macular edema disappeared in half of the group) was 12 months (range 1-24 months) (Fig. 2b). Older age at the time of RVO (HR $=0.88,95 \%$ CI 0.79-0.99, $p=0.03$ ) and BRVO $\quad(\mathrm{HR}=0.12,95 \%$ CI $0.01-0.92$, $p=0.04)$ were risk factors for persistent macular edema after ERM removal (Table 4). 



Fig. 1 Best-corrected visual acuity (BCVA, expressed as logMAR, a) and central macular thickness (CMT, expressed as $\mu \mathrm{m}$, b) change as a function of the follow-up visit after epiretinal membrane removal



B

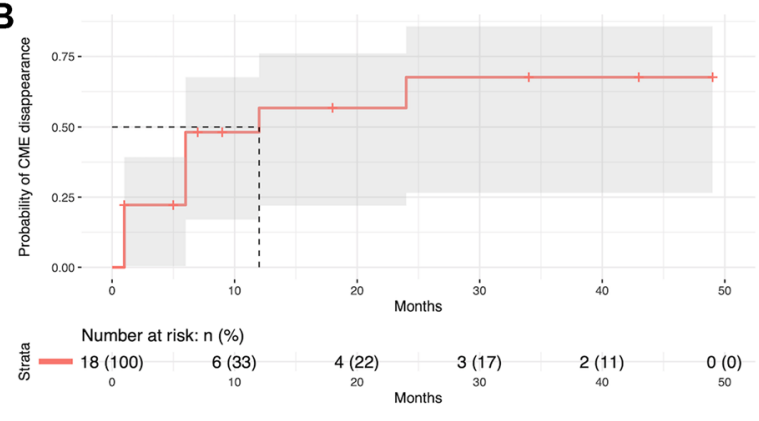

Fig. 2 Kaplan-Meier survival curves showing the probability of one-line improvement in best-corrected visual acuity (BCVA) and disappearance of cystoid macular edema (CME) after epiretinal membrane removal. a Cumulative probability of one-line BCVA improvement. The time at which the Kaplan-Meier survival curve crossed the $50 \%$ probability line (i.e., the median survival time) was 1 month. b Cumulative probability of CME disappearance. The median survival time was 12 months
Multivariable analyses were not performed due to low numbers.

Additional anti-VEGF agents were administered in six cases (29\%); the median number of injections was 1 , statistically significantly lower than before PPV $(p<0.02)$. Additional DEX implants were administered in 12 eyes $(57 \%$, median injection number was 2). The number of treatments given after ERM removal was not associated with the probability of macular edema disappearance after surgery (Table 3).

\section{Longitudinal Safety}

Two eyes underwent cataract extraction after PPV. Recurrent ERM was seen in four eyes (19\%), but none underwent a second surgery during the follow-up. ERM recurrence was not visually impairing ( $p=0.2$, Table 2$)$, was not associated with significant retinal thickening $(p=0.8$, Table 3$)$, and was not a risk factor for persistent macular edema after primary ERM removal $(p=0.3$, Table 4$)$.

\section{DISCUSSION}

In the present study, PPV + membrane removal in eyes with ERM secondary to RVO led to significant improvement in visual acuity and macular thickening, with disappearance of macular edema in most eyes. Disruption the of 
Table 3 Results of univariable analysis of factors associated with the longitudinal change in best-corrected visual acuity (BCVA) and central macular thickness (CMT) after epiretinal membrane (ERM) removal in eyes with retinal vein occlusion (RVO)

Variable

BCVA change

\section{Regression estimate} (95\% CI) [logMAR]

Patient characteristics

Age at RVO (for each year)

Male gender (ref: female)

Diabetes

Cardiovascular risk factors

Glaucoma

Surgery characteristics

Cataract extraction ${ }^{\wedge}$

PVD induction

ILM peeling

Gas tamponade

Eye characteristics

BRVO (ref: CRVO)

Interval RVO to ERM peeling (for each

12 months)

ELM/EZ loss before ERM removal

ELM/EZ loss after ERM removal

DRIL before ERM removal

DRIL after ERM removal

Peripheral laser photocoagulation

Number of anti-VEGF doses administered before ERM removal (for each injection)

Number of anti-VEGF doses administered after ERM removal (for each injection)

Number of DEX implants administered before ERM removal (for each injection)

Number of DEX implants administered after ERM removal (for each injection)

$$
\begin{array}{rlccc}
-0.01(-0.04 \text { to } 0.01) & 0.4 & 3.5(-2.5 \text { to } 9.4) & 0.3 \\
-0.01(-0.43 \text { to } 0.41) & 0.9 & -21.2(-135.4 \text { to } 93.2) & 0.7 \\
-0.03(-0.67 \text { to } 0.62) & 0.9 & -29.7(-192.6 \text { to } 132.8) & 0.7 \\
0.28(-0.15 \text { to } 0.70) & 0.2 & 42.3(-74.3 \text { to } 159.4) & 0.2 \\
-0.49(-0.90 \text { to } 0.06) & 0.05 & -121.9(-239.2 \text { to } 4.8) & 0.06
\end{array}
$$

$$
\begin{array}{rrrr}
0.34(-0.09 \text { to } 0.76) & 0.1 & -76.4(-189.4 \text { to } 36.8) & 0.2 \\
-0.07(-0.58 \text { to } 0.39) & 0.8 & 106.5(-11.1 \text { to } 224.2) & 0.1 \\
-0.37(-0.95 \text { to } 0.20) & 0.2 & 91(-67.5 \text { to } 249.7) & 0.3 \\
0.15(-0.37 \text { to } 0.69) & 0.6 & 78.8(-64.1 \text { to } 221.3) & 0.3
\end{array}
$$

$$
\begin{array}{llcll}
-0.29(-0.71 \text { to } 0.14) & 0.2 & 74.1(-42.5 \text { to } 191.1) & 0.2 \\
-0.03(-0.08 \text { to } 0.02) & 0.2 & 2.3(-11.6 \text { to } 16.2) & 0.8
\end{array}
$$

\begin{tabular}{ccccc}
$0.87(0.53$ to 1.22$)$ & $<0.001^{*}$ & $128.3(-4.19$ to 252.5$)$ & 0.06 \\
$0.99(0.62$ to 1.35$)$ & $<0.001^{*}$ & $69.9(-58.1$ to 197.9$)$ & 0.3 \\
$0.09(-0.34$ to 0.50$)$ & 0.7 & $114.1(78.9$ to 219.4$)$ & $0.04^{*}$ \\
$0.68(0.22$ to 1.14$)$ & $0.009^{*}$ & $217.5(91.7$ to 342.7$)$ & $0.002^{*}$ \\
$-0.02(-0.44$ to 0.40$)$ & 0.9 & $59.3(-58.4$ to 177.9$)$ & 0.3 \\
$-0.01(-0.50$ to 0.03$)$ & 0.7 & $-5.8(-15.1$ to 3.40$)$ & 0.3 \\
$-0.01(-0.14$ to 0.17$)$ & 0.9 & $-40.7(-60.8$ to -21.5$)$ & 0.08 \\
$-0.12(-0.30$ to 0.05$)$ & 0.2 & $-24.5(-64.1$ to 14.9$)$ & 0.2 \\
$-0.08(-0.23$ to 0.05$)$ & 0.3 & $-27.7(-67.2$ to 12.5$)$ & 0.2 \\
\hline
\end{tabular}


Table 3 continued

\begin{tabular}{|c|c|c|c|c|}
\hline \multirow[t]{2}{*}{ Variable } & \multicolumn{2}{|l|}{ BCVA change } & \multicolumn{2}{|l|}{ CMT change } \\
\hline & $\begin{array}{l}\text { Regression estimate } \\
(95 \% \mathrm{CI})[\log \mathrm{AAR}]\end{array}$ & $p$ value & $\begin{array}{l}\text { Regression estimate }(95 \% \\
\text { CI) }[\mu \mathrm{m}]\end{array}$ & $p$ value \\
\hline ERM recurrence & $0.39(-0.19$ to 0.93$)$ & 0.2 & $12.9(-132.8$ to 158.3$)$ & 0.8 \\
\hline
\end{tabular}

Regression estimates and the $95 \%$ confidence interval (CI) are presented

PVD posterior vitreous detachment, $I L M$ inner limiting membrane, BRVO branch retinal vein occlusion, $C R V O$ central retinal vein occlusion, $E L M / E Z$ external limiting membrane/ellipsoid zone, DRIL disorganization of the retinal inner layers, $V E G F$ vascular endothelial growth factor, $D E X$ dexamethasone

$\wedge$ Refers to eyes that were phakic at the time of the surgery

*statistically significant value $(\mathrm{p}<0.05)$

EZ/ELM and persistence of DRIL after ERM peeling were associated with worse visual outcomes, while the presence of DRIL at baseline and persistence of DRIL on follow-up were associated with worse anatomical results. Older patients and those with BRVO had a higher risk of persistent macular thickening after surgery (Fig. 3).

Both primary and secondary ERMs have two main components: an extracellular matrix (consisting of collagen, laminin, and fibronectin) and cells of retinal and extraretinal origin, such as glial cells, neurites, retinal pigment epithelium, immune cells, and fibrocytes $[2,17,18]$. The relative abundance of these components within each ERM case reflects the underlying etiology and the severity of the disease or its duration. In retinal ischemia or inflammation, such as in diabetic retinopathy or RVO, activation of Müller cells induces the upregulation of glial fibrillary acidic protein and vimentin, with reactive gliosis and ERM formation $[19,20]$. As platelet-derived growth factor A (PDGF A) and VEGF receptors are expressed within ERM cells, intravitreal proangiogenic cytokines may accelerate ERM progression [21]. Contractile fibrils eventually exert traction on the underlying retina and distort the retinal tissue and the retinal vasculature, causing visual loss [22]. Removal of ERM may revert these processes, provided that inner and outer retinal integrity is preserved.

In our study, nearly $80 \%$ of eyes had a history of previous intravitreal injections. Intravitreal injections have been hypothesized to contribute to ERM formation. A higher number of intravitreal injections of anti-VEGF and the use of DEX implants have been associated with higher odds of ERM in patients with diabetic macular edema [23]. Possible effects of intravitreal VEGF inhibition include an increase of retinal ischemia, an imbalance towards the effect of pigment epithelium-derived factor, or an indirect increase in the expression of connective tissue growth factor, which stimulates tissue fibrosis and ERM formation [24]. On the other hand, intravitreal DEX implant has been thought to induce mechanical stress into the vitreous cavity, leading to alteration in the vitreomacular interface homeostasis and epiretinal tissue proliferation [25]. We cannot exclude the possibility that intravitreal therapies contributed to the pathogenesis of ERM in our RVO patients. Nevertheless, we did not include a control group of RVO patients without ERM, and we were not able to verify this hypothesis.

The improvement in visual acuity after PPV and ERM peeling was rapid and sustained over time. It might be argued that spontaneous visual recovery is likely to occur in the natural history of RVO [26]. In the SCORE study, up to $26 \%$ of eyes in the sham group experienced a visual gain ranging from 5 to 15 letters, although no data were provided specifically about eyes with ERM [27]. However, visual acuity gains tend to stabilize between the second and fifth year of treatment [28]. Since the median interval between RVO diagnosis and PPV was 3 years, our data may support an actual advantage of ERM peeling on the visual 
Table 4 Factors associated with macular edema disappearance after epiretinal membrane (ERM) removal in eyes with retinal vein occlusion (RVO)

\begin{tabular}{|c|c|c|}
\hline & HR (95\% CI) & $p$ value \\
\hline Age at RVO (for each year) & $0.88(0.79$ to 0.99$)$ & $0.03^{*}$ \\
\hline Male gender (ref: female) & $4.44(0.92$ to 21.5$)$ & 0.06 \\
\hline Diabetes & $0.82(0.10$ to 6.58$)$ & 0.8 \\
\hline Cardiovascular risk factors & $0.61(0.15$ to 2.46$)$ & 0.5 \\
\hline Glaucoma & $0.8(0.17$ to 4.04$)$ & 0.8 \\
\hline \multicolumn{3}{|l|}{ Surgery characteristics } \\
\hline Cataract extraction ${ }^{\wedge}$ & $0.34(0.09$ to 0.76$)$ & 0.3 \\
\hline PVD induction & $0.71(0.18$ to 2.88$)$ & 0.6 \\
\hline ILM peeling & $1.31(0.16$ to 10.53$)$ & 0.7 \\
\hline Gas tamponade & $1.50(0.31$ to 7.21$)$ & 0.6 \\
\hline \multicolumn{3}{|l|}{ Eye characteristics } \\
\hline BRVO (ref: CRVO) & $0.12(0.01$ to 0.92$)$ & $0.04^{*}$ \\
\hline Interval RVO to ERM peeling (for each 12 months) & $1.00(0.98$ to 1.02$)$ & 0.7 \\
\hline Peripheral laser photocoagulation & $1.82(0.54$ to 6.60$)$ & 0.3 \\
\hline ELM/EZ loss before ERM removal & $0.69(0.17$ to 2.75$)$ & 0.2 \\
\hline ELM/EZ loss after ERM removal & $0.17(0.02$ to 1.41$)$ & 0.1 \\
\hline DRIL before ERM removal & $0.66(1.19$ to 2.34$)$ & 0.5 \\
\hline DRIL after ERM removal & $0.58(0.12$ to 2.77$)$ & 0.5 \\
\hline Number of anti-VEGF doses administered before ERM removal (for each injection) & $1.02(0.86$ to 1.21$)$ & 0.8 \\
\hline Number of anti-VEGF doses administered after ERM removal (for each injection) & $1.51(0.75$ to 3.05$)$ & 0.8 \\
\hline Number of DEX implants administered before ERM removal (for each injection) & $0.95(0.58$ to 1.57$)$ & 0.2 \\
\hline Number of DEX implants administered after ERM removal (for each injection) & $0.91(0.60$ to 1.39$)$ & 0.7 \\
\hline ERM recurrence & $0.40(0.08$ to 2.06$)$ & 0.3 \\
\hline
\end{tabular}

Hazard ratio and the $95 \%$ confidence interval (CI) are presented from univariable analysis

PVD posterior vitreous detachment, $I L M$ inner limiting membrane, $B R V O$ branch retinal vein occlusion, $C R V O$ central retinal vein occlusion, $E L M / E Z$ external limiting membrane/ellipsoid zone, DRIL disorganization of the retinal inner layers, $V E G F$ vascular endothelial growth factor, $D E X$ dexamethasone

${ }^{\wedge}$ Refers to eyes that were phakic at the time of the surgery

${ }^{*}$ statistically significant value $(\mathrm{p}$ value $<0.05)$

outcomes. The benefits of PPV with ERM may be attributed to the following mechanisms: the elimination of vitreous traction on the macular area, the increase of oxygen diffusion into the retina, and the removal of pro-angiogenic mediators from the vitreous chamber [29].
Moreover, the removal of ERM may increase the drug penetration into the retina after intravitreal injections $[7,8]$. A beneficial effect of ILM peeling during vitrectomy for RVO has also been correlated with decompression of the edematous retina [30], even though ILM peeling 


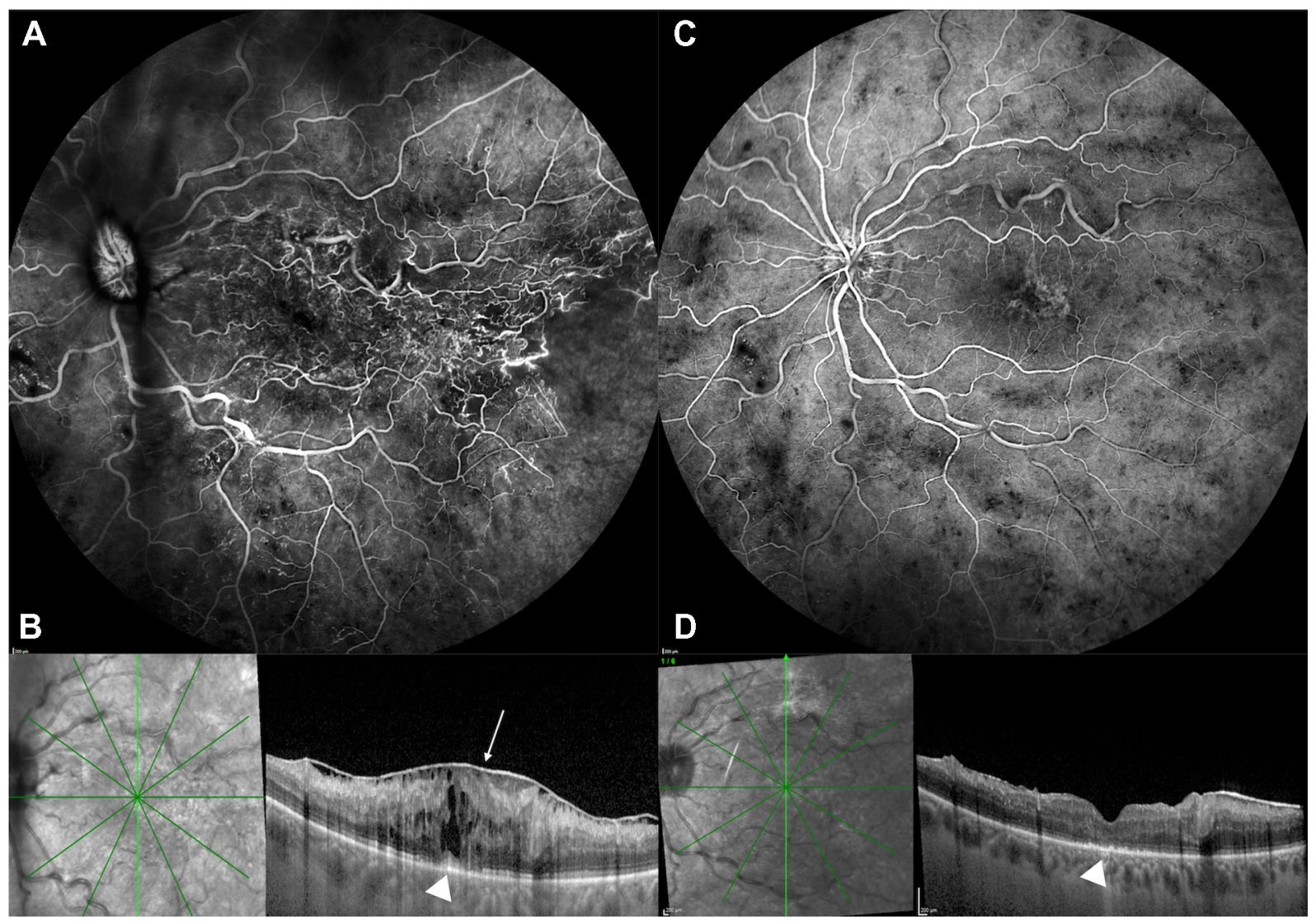

Fig. 3 Clinical follow-up of a patient with central retinal vein occlusion before and after epiretinal membrane (ERM) removal. a Fluorescein angiography (FA) showed marked vascular tortuosity and vessel dragging temporally to the fovea, as well as enlargement of the foveal avascular zone and temporal non-perfusion. b Optical coherence tomography (OCT) corresponding to the same visit as a showing macular edema, intraretinal cysts, disruption of

was not found to be significantly associated with treatment outcomes in our cohort.

The state of the EZ and ELM is an independent prognostic factor for visual outcome after intravitreal injections in patients with RVO $[31,32]$. Moreover, EZ/ELM integrity has been associated with better visual outcomes in patients with idiopathic ERM after PPV and ERM removal [33]. Kang et al. investigated the factors correlating with visual acuity after surgery and ERM peeling in 33 eyes with BRVO. The authors identified integrity of photoreceptors at baseline as a predictor of the visual outcome in these patients [12]. In keeping with the external limiting membrane/ellipsoid zone complex (arrowhead), and a thick ERM (arrow). c FA performed 3 months after ERM peeling, showing partial normalization of the vascular network. There was persistence of foveal avascular zone enlargement and mild leakage temporally to the fovea. $\mathbf{d}$ OCT disclosed a flat macula, with restoration of the foveal depression. External limiting membrane/ ellipsoid zone complex defects persisted (arrowhead)

this, our study showed that disruption of the EZ/ELM at the time of ERM removal was associated with worse visual acuity for the entire follow-up after the surgery, up to 36 months. Persistent DRIL after ERM removal was also associated with worse anatomical and visual outcomes. The presence of DRIL indirectly suggests disruption of the visual transmission pathway, which may have multiple interpretations in eyes with RVO [15]. It may be caused by poor macular perfusion or by tractional distortion of the retinal layers. Of note, DRIL has been identified as an adverse prognostic factor after idiopathic ERM removal [13, 34]. Our study 
supports the negative impact of DRIL on visual recovery in eyes with ischemic retinopathies.

The visual outcome after ERM peeling was similar between CRVO and BRVO. However, eyes with BRVO had persistent macular edema after surgery. Higher levels of inflammatory markers and VEGF-A have been found in eyes with CRVO by comparing the undiluted vitreous samples of these eyes with those diagnosed with BRVO [35]. It is plausible that removing the vitreous scaffold and its cytokines would have a greater impact on CRVO eyes than BRVO ones. On the other hand, BRVO eyes might have a tighter ERM due to the presence of epiretinal neovascularization, much more prevalent in BRVO than CRVO eyes [36]. Due to the lack of fluorescein angiography data, we were not able to include the presence of epiretinal neovascularization in our models.

Limitations of this study include the small study sample and its retrospective design. As the patients were included from different centers and they were treated by different physicians, we acknowledge possible heterogeneity in treatments' regimens, preference in choosing the intravitreal drugs, and criteria to send patients to retinal surgery. We did not include other imaging modalities, such as OCT angiography and widefield fluorescein angiography, which differentiate between ischemic and nonischemic RVOs. The study patients underwent a slightly lower number of anti-VEGF injections compared to other real-life series. Some eyes with evident ERM might have received less intense treatment, given the tractional nature of the retinal thickening. Our study patients also underwent DEX injections; this could be an additional factor explaining the low number of anti-VEGF injections administered before ERM peeling. We did not assess other vitreomacular disorders, such as vitreomacular traction, which are important factors in the decisional algorithm of vitreoretinal surgeons. Due to the low numbers, we acknowledge the risk of overfitting our models and underpowering the explanatory variables; larger studies are needed to confirm our associations. Finally, a control group of eyes that did not require pharmacological treatment post-ERM peeling could throw light on the effect of ERM peeling on macular edema resolution. Nevertheless, ERM peeling in RVO eyes is uncommon, and controlled retrospective studies are barely feasible.

\section{CONCLUSIONS}

PPV and ERM removal provided encouraging functional and morphological results in eyes with RVO. Integrity of the outer retina and preservation of inner retinal segmentation were associated with better visual and anatomical outcomes after ERM removal, respectively. History of CRVO was associated with a higher chance of macular anatomical restoration. Further prospective, controlled studies with a larger study sample are needed to validate our findings.

\section{ACKNOWLEDGEMENTS}

Authors do thank all study participants for their involvement in the study.

Funding. No funding or sponsorship was received for this study or publication of this article.

Authorship. All named authors meet the International Committee of Medical Journal Editors (ICMJE) criteria for authorship for this article, take responsibility for the integrity of the work as a whole, and have given their approval for this version to be published.

Author Contributions. Conceptualization: Maria Vittoria Cicinelli, Irini Chatziralli. Methodology: Sara Touhami, Marco Nassisi. Data collection: Anissa Smaoui, Marco Nassisi, Maria Vittoria Cicinelli, Irini Chatziralli. Visualization, Data curation: Beatrice Tombolini. Writing - Original draft preparation: Maria Vittoria Cicinelli, Irini Chatziralli. Writing - Review and Editing: Sara Touhami, Marco Nassisi. Supervision and project administration: Panagiotis Theodossiadis, Rosangela Lattanzio, Francesco Bandello. 
Disclosures. The Authors have no competing interest in publishing the present work. Maria Vittoria Cicinelli, Beatrice Tombolini, Marco Nassisi, Anissa Smaoui, Panagiotis Theodossiadis: none. Irini Chatziralli: travel grants and consultant fees from Novartis (Basel, Switzerland), Bayer Schering-Pharma (Berlin, Germany), Allergan (Irvine, California, USA), Thea-Laboratories, Novo-Nordisk, Innovis Pharma. Sarah Touhami: travel grants and consultant fees from Allergan Inc (Irvine, California, USA), Novartis (Basel, Switzerland), Bayer Schering-Pharma (Berlin, Germany). Rosangela Lattanzio: consultant for Allergan Inc (Irvine, California, USA), Novartis (Basel, Switzerland), Bayer Schering-Pharma (Berlin, Germany), Sifi (Aci Sant'Antonio, Italy). Francesco Bandello: consultant for Allergan Inc (Irvine, California, USA), Bayer ScheringPharma (Berlin, Germany), Hoffmann-La-Roche (Basel, Switzerland), Novartis (Basel, Switzerland), Sanofi-Aventis (Paris, France), Thrombogenics (Heverlee, Belgium), Zeiss (Dublin, USA), Boehringer-Ingelheim, Fidia Sooft, Ntc Pharma, Sifi (Aci Sant'Antonio, Italy).

Compliance with Ethics Guidelines. The study adhered to the tenets of the Declaration of Helsinki (1964) and received the approval of the local institutional review boards. Patients signed a consent for retrospective clinical studies.

Data Availability. Data are available upon request to the corresponding author.

Open Access. This article is licensed under a Creative Commons Attribution-NonCommercial 4.0 International License, which permits any non-commercial use, sharing, adaptation, distribution and reproduction in any medium or format, as long as you give appropriate credit to the original author(s) and the source, provide a link to the Creative Commons licence, and indicate if changes were made. The images or other third party material in this article are included in the article's Creative Commons licence, unless indicated otherwise in a credit line to the material. If material is not included in the article's Creative Commons licence and your intended use is not permitted by statutory regulation or exceeds the permitted use, you will need to obtain permission directly from the copyright holder. To view a copy of this licence, visit http://creativecommons.org/licenses/by$\mathrm{nc} / 4.0 /$.

\section{REFERENCES}

1. Bu SC, Kuijer R, Li XR, et al. Idiopathic epiretinal membrane. Retina. 2014;34(12):2317-35. https:// doi.org/10.1097/IAE.0000000000000349.

2. Sandali O, El Sanharawi M, Basli E, et al. Epiretinal membrane recurrence: incidence, characteristics, evolution, and preventive and risk factors. Retina. 2013;33(10):2032-8. https://doi.org/10.1097/IAE. 0b013e31828d2fd6.

3. McIntosh RL, Rogers SL, Lim L, et al. Natural history of central retinal vein occlusion: an evidence-based systematic review. Ophthalmology. 2010;117(6): 1113-1123.e1115. https://doi.org/10.1016/j. ophtha.2010.01.060.

4. Fraser-Bell S, Ying-Lai M, Klein R, et al. Prevalence and associations of epiretinal membranes in latinos: the Los Angeles Latino Eye Study. Invest Ophthalmol Vis Sci. 2004;45(6):1732-6. https://doi. org/10.1167/iovs.03-1295.

5. Duan XR, Liang YB, Friedman DS, et al. Prevalence and associations of epiretinal membranes in a rural Chinese adult population: the Handan Eye Study. Invest Ophthalmol Vis Sci. 2009;50(5):2018-23. https://doi.org/10.1167/iovs.08-2624.

6. Mitchell P, Smith W, Chey T, et al. Prevalence and associations of epiretinal membranes. The Blue Mountains Eye Study, Australia. Ophthalmology. 1997;104(6):1033-40. https://doi.org/10.1016/ s0161-6420(97)30190-0.

7. Hwang S, Kang SW, Kim KT, et al. Three-year outcomes of vitrectomy combined with intraoperative dexamethasone implantation for non-tractional refractory diabetic macular edema. Sci Rep. 2021;11(1):1292. https://doi.org/10.1038/s41598020-80350-w.

8. Ghassemi F, Bazvand F, Roohipoor R, et al. Outcomes of vitrectomy, membranectomy and internal limiting membrane peeling in patients with refractory diabetic macular edema and non-tractional epiretinal membrane. J Curr Ophthalmol. 2016;28(4):199-205. https://doi.org/10.1016/j.joco. 2016.08.006. 
9. Coassin M, Mori T, Mastrofilippo V, et al. Surgical management of post-uveitic epiretinal membranes. Eur J Ophthalmol. 2021. https://doi.org/10.1177/ 11206721211017750.

10. Assi A, Chacra CB, Cherfan G. Combined lensectomy, vitrectomy, and primary intraocular lens implantation in patients with traumatic eye injury. Int Ophthalmol. 2008;28(6):387-94. https://doi. org/10.1007/s10792-007-9151-9.

11. DeCroos FC, Shuler RK Jr, Stinnett S, Fekrat S. Pars plana vitrectomy, internal limiting membrane peeling, and panretinal endophotocoagulation for macular edema secondary to central retinal vein occlusion. Am J Ophthalmol. 2009;147(4):627-633. e621. https://doi.org/10.1016/j.ajo.2008.10.024.

12. Kang HM, Koh HJ, Lee SC. Visual outcome and prognostic factors after surgery for a secondary epiretinal membrane associated with branch retinal vein occlusion. Graefes Arch Clin Exp Ophthalmol. 2015;253(4):543-50. https://doi.org/10.1007/ s00417-014-2731-2.

13. Zur D, Iglicki M, Feldinger L, et al. Disorganization of retinal inner layers as a biomarker for idiopathic epiretinal membrane after macular surgery-the DREAM study. Am J Ophthalmol. 2018;196:129-35. https://doi.org/10.1016/j.ajo.2018.08.037.

14. Chan EW, Eldeeb M, Sun V, et al. Disorganization of retinal inner layers and ellipsoid zone disruption predict visual outcomes in central retinal vein occlusion. Ophthalmol Retina. 2019;3(1):83-92. https://doi.org/10.1016/j.oret.2018.07.008.

15. Mimouni M, Segev O, Dori D, et al. Disorganization of the retinal inner layers as a predictor of visual acuity in eyes with macular edema secondary to vein occlusion. Am J Ophthalmol. 2017;182:160-7. https://doi.org/10.1016/j.ajo.2017.08.005.

16. Lange C, Feltgen N, Junker B, et al. Resolving the clinical acuity categories "hand motion" and "counting fingers" using the Freiburg Visual Acuity Test (FrACT). Graefes Arch Clin Exp Ophthalmol. 2009;247(1):137-42. https://doi.org/10.1007/ s00417-008-0926-0.

17. Lesnik Oberstein SY, Lewis GP, Dutra T, Fisher SK. Evidence that neurites in human epiretinal membranes express melanopsin, calretinin, rod opsin and neurofilament protein. $\mathrm{Br} \mathrm{J}$ Ophthalmol. 2011;95(2):266-72. https://doi.org/10.1136/bjo. 2010.180679 .

18. Mackenzie SE, Gandorfer A, Rohleder $\mathrm{M}$, et al. Ultrastructure and retinal imaging of epiretinal membrane: a clinicopathologic correlation of trypan blue staining in epiretinal membrane surgery.
Retina. 2010;30(4):648-54. https://doi.org/10. 1097/IAE.0b013e3181bceda9.

19. Lee SY, Surbeck JW, Drake M, et al. Increased glial fibrillary acid protein and vimentin in vitreous fluid as a biomarker for proliferative vitreoretinopathy. Invest Ophthalmol Vis Sci. 2020;61(5):22. https:// doi.org/10.1167/iovs.61.5.22.

20. Romano MR, Ilardi G, Ferrara M, et al. Intraretinal changes in idiopathic versus diabetic epiretinal membranes after macular peeling. PLoS ONE. 2018;13(5): e0197065. https://doi.org/10.1371/ journal.pone.0197065.

21. Chen YS, Hackett SF, Schoenfeld CL, et al. Localisation of vascular endothelial growth factor and its receptors to cells of vascular and avascular epiretinal membranes. Br J Ophthalmol. 1997;81(10): 919-26. https://doi.org/10.1136/bjo.81.10.919.

22. Romano MR, Cennamo G, Schiemer S, et al. Deep and superficial OCT angiography changes after macular peeling: idiopathic vs diabetic epiretinal membranes. Graefes Arch Clin Exp Ophthalmol. 2017;255(4):681-9. https://doi.org/10.1007/ s00417-016-3534-4.

23. Kang YK, Park HS, Park DH, Shin JP. Incidence and treatment outcomes of secondary epiretinal membrane following intravitreal injection for diabetic macular edema. Sci Rep. 2020;10(1):528. https:// doi.org/10.1038/s41598-020-57509-6.

24. Marticorena J, Romano MR, Heimann $\mathrm{H}$, et al. Intravitreal bevacizumab for retinal vein occlusion and early growth of epiretinal membrane: a possible secondary effect? Br J Ophthalmol. 2011;95(3): 391-5. https://doi.org/10.1136/bjo.2009.177287.

25. Panjaphongse R, Stewart JM. Vitreomacular traction after dexamethasone intravitreal implant (ozurdex) injection: the effect of anomalous posterior vitreous detachment. Retin Cases Brief Rep. 2016;10(1):55-7. https://doi.org/10.1097/ICB. 0000000000000172 .

26. Hayreh SS, Podhajsky PA, Zimmerman MB. Natural history of visual outcome in central retinal vein occlusion. Ophthalmology. 2011;118(1):119-133. e111-112. https://doi.org/10.1016/j.ophtha.2010. 04.019.

27. Ip MS, Scott IU, VanVeldhuisen PC, et al. A randomized trial comparing the efficacy and safety of intravitreal triamcinolone with observation to treat vision loss associated with macular edema secondary to central retinal vein occlusion: the Standard Care vs Corticosteroid for Retinal Vein Occlusion (SCORE) study report 5. Arch Ophthalmol. 2009;127(9):1101-14. https://doi.org/10. 1001/archophthalmol.2009.234. 
28. Spooner K, Fraser-Bell S, Hong T, Chang AA. Fiveyear outcomes of retinal vein occlusion treated with vascular endothelial growth factor inhibitors. BMJ Open Ophthalmol. 2019;4(1): e000249. https://doi. org/10.1136/bmjophth-2018-000249.

29. Diabetic Retinopathy Clinical Research Network Writing C, Haller JA, Qin H, et al. Vitrectomy outcomes in eyes with diabetic macular edema and vitreomacular traction. Ophthalmology. 2010;117(6):1087-1093.e1083. https://doi.org/10. 1016/j.ophtha.2009.10.040.

30. Mandelcorn MS, Nrusimhadevara RK. Internal limiting membrane peeling for decompression of macular edema in retinal vein occlusion: a report of 14 cases. Retina. 2004;24(3):348-55. https://doi. org/10.1097/00006982-200406000-00002.

31. Chatziralli I, Theodossiadis G, Chatzirallis A, et al. Ranibizumab for retinal vein occlusion: predictive factors and long-term outcomes in real-life data. Retina. 2018;38(3):559-68. https://doi.org/10. 1097/IAE.0000000000001579.

32. Battaglia Parodi M, Iacono P, Scaramuzzi M, Bandello F. Outer retinal layer changes after dexamethasone implant for central retinal vein occlusion.
Retina. 2017;37(10):1888-95. https://doi.org/10. 1097/IAE.0000000000001429.

33. Chatziralli I, Dimitriou E, Xirou T, et al. Optical coherence tomography biomarkers as predictive factors for postoperative visual acuity in patients with epiretinal membrane treated with vitrectomy. Oman J Ophthalmol. 2020;13(3):136-40. https:// doi.org/10.4103/ojo.OJO_79_2020.

34. Garnavou-Xirou C, Xirou T, Gkizis I, et al. The role of disorganization of retinal inner layers as predictive factor of postoperative outcome in patients with epiretinal membrane. Ophthalmic Res. 2020;63(1):13-7. https://doi.org/10.1159/ 000499370 .

35. Koss MJ, Pfister M, Rothweiler F, et al. Comparison of cytokine levels from undiluted vitreous of untreated patients with retinal vein occlusion. Acta Ophthalmol. 2012;90(2):e98-103. https://doi.org/ 10.1111/j.1755-3768.2011.02292.x.

36. Hayreh SS, Rojas P, Podhajsky P, et al. Ocular neovascularization with retinal vascular occlusion-III. Incidence of ocular neovascularization with retinal vein occlusion. Ophthalmology. 1983;90(5): 488-506. $6420(83) 34542-5$. 